\title{
Gouverner par les morts et les mots. Quand le passé colonial et la « culture » immigrée deviennent une priorité municipale
}

Governing through the Dead and the Words: When Colonial History and Immigrant "Culture" Become a Political Priority

\section{Victor Collet}

\section{(2) OpenEdition Journals}

\section{Édition électronique}

URL : http://journals.openedition.org/conflits/19550

DOI : 10.4000/conflits. 19550

ISSN : $1777-5345$

\section{Éditeur :}

CCLS - Centre d'études sur les conflits lilberté et sécurité, L'Harmattan

\section{Édition imprimée}

Date de publication : 30 octobre 2017

Pagination : 77-103

ISBN : 978-2-343-13540-3

ISSN : $1157-996 X$

\section{Référence électronique}

Victor Collet, « Gouverner par les morts et les mots. Quand le passé colonial et la « culture » immigrée deviennent une priorité municipale », Cultures \& Conflits [En ligne], 107 | automne 2017, mis en ligne le 30 octobre 2019, consulté le 30 mars 2021. URL : http://journals.openedition.org/conflits/19550 ; DOI : https://doi.org/10.4000/conflits. 19550 


\section{Gouverner par les morts et les mots. Quand le passé colonial et la " culture» immigrée deviennent une priorité municipale}

\section{Victor COLLET}

Victor Collet est docteur en science politique (Labex "Les passés dans le présent "/Institut des sciences sociales du politique, Nanterre). Auteur de: Nanterre, du bidonville à la cité, Marseille, Agone, à paraître (janvier 2018); "Un soutien critique : le Comité Gutenberg (Nanterre) et la Marche pour l'égalité de 1983 ", in Hadj B., Nasri S. (dir.), La Marche de 1983 : des mémoires à l'histoire d'une mobilisation collective, Nanterre, Presses Universitaires de Nanterre, à paraître (2018); "Entre ressource intellectuelle et cause politique. Les différentes vies d'Abdelmalek Sayad », Politix, n94, 2011, pp. 155-178.

Un acte de mémoire et d'avenir

La date du 17 octobre 1961 appartient à notre histoire. [...] Aujourd'bui encore, cinquante ans après les faits, le crime n'est toujours pas reconnu et la plupart de ses victimes restent anonymes. Si la ville de Nanterre a décidé de s'impliquer activement dans le cinquantième anniversaire de ce drame, c'est pour que l'ensemble de notre population, au-delà d'origines et de parcours différents, s'approprie cette part de notre histoire commune. La décision $d u$ conseil municipal [...] marque la reconnaissance d'une ville riche de tous ses ailleurs, elle contribue au "vivre ensemble".

Patrick Jarry

« 17 octobre 1961. Nanterre ne veut pas oublier », Brochure du cinquantenaire du 17 octobre 1961, octobre 2011 

octobre 2011. Esplanade de la Défense. Les maires de plusieurs communes de l'ancienne boucle nord-ouest de la Seine sont réunis face au Pont de Neuilly en présence de plusieurs centaines de personnes. Suite au refus du maire de Neuilly-sur-Seine d'accueillir l'événement, les élus commémorent au milieu des tours d'affaires le massacre des centaines d'Algériens tués par la police parisienne lors de la manifestation du 17 octobre 1961. Nombre d'entre eux, venus des banlieues environnantes, étaient alors stoppés par les balles de la police en tentant de rejoindre Paris. Cinquante ans plus tard, les célébrations dureront près d'un mois dans certaines communes de l'ancienne " ceinture rouge ", faisant revivre ces indénombrables noyés par balle ${ }^{1} \mathrm{du}$ « 17 octobre ». Officielles, culturelles ou même festives, les cérémonies s'adressent alors aux vivants et aux générations postérieures d'enfants de l'immigration et de la banlieue. Banlieue qu'elles débordent : de nombreux élus nationaux rejoignent ainsi, ce 17 octobre, le défilé parisien qui descend les Grands boulevards. Accompagné de milliers de manifestants, il se clôt au Pont Saint-Michel où une plaque commémorative a été posée en 2001.

2011 constitue une sorte d'apogée du souvenir du 17 octobre 1961 et de son institutionnalisation. Or, l'investissement simultané de plusieurs municipalités de gauche «plurielle» interroge. Il impose de revenir sur la décennie écoulée et la soudaine ascension des mémoires coloniales et «immigrées » à l'état de politiques publiques et institutionnelles. Le souvenir de la cité rouge s'estompe devant celui des bidonvilles. Difficile dans le cas de Nanterre d'avancer l'hypothèse d'un effet soudain d'une transformation sociographique liée à l'arrivée de migrants qui modifierait le jeu politique. Depuis au moins les années 1950, la municipalité est objectivement une ville d'accueil et voit son capital symbolique associé à l'histoire des migrations. Mais ce qui change est sans doute le caractère volontariste dans la revendication publique de cet héritage. Semblable en cela à d'autres villes de la ceinture parisienne comme Montreuil 2, la mairie de Nanterre revendique désormais plus volontiers ce pan de son histoire politique que son héritage communiste. Le maintien des dirigeants en place, la perpétuation d'une certaine endocratie ${ }^{3}$ familiale ou partisane impose des redirections. Face à l'effacement des bases du communisme et du socialisme municipaux, renouveler les cadres et les légitimités qui façonnaient la stabilité politique municipale d'hier est devenu une nécessité.

La longue traversée du désert des relations entre histoire coloniale, « logement étranger » et gauche municipale laisserait ainsi place à la sanctuarisation d'événements à la mémoire de l'immigration, à un nouveau devoir de mémoire

1. House J., MacMaster N., Paris 1961. Les Algériens, la terreur d’État et la mémoire, Paris, Tallandier, 2008, p. 24.

2. Palomares E., Les Paradoxes de la préférence locale : engagements collectifs et action publique à l'éprenve de l'ethnicisation des rapports sociaux. Montreuil, Seine-Saint-Denis, Thèse pour le doctorat de sociologie, dir. Quiminal C., Paris 7, 2003. 
coloniale et immigrée. Le 17 octobre 1961 en représente le parangon le plus commun ou communalisé : national, exceptionnel et hautement dramatique, il imprime cette nouvelle ère municipale qui ne fait plus table rase du passé et des morts de la colonisation. Les temps semblent révolus qui inspiraient au poète antillais Ludovic Janvier le piquant «Vous me parlez d'octobre 17, moi je vous parle du 17 octobre 4 ». Reste pourtant à saisir ce que ce brutal changement du regard sur l'immigration 5 dit des banlieues populaires de l'après 2005. Et à apprécier comment ce lent processus de déstigmatisation, circonscrit et localisé, s'inscrit dans une configuration générale de catégorisation négative des «immigrés » et d'opposition récurrente à leur intégration à une hypothétique identité nationale ${ }^{6}$.

C'est en ce sens que le retour au local prévaut dans cet article, comme il a prévalu dans la recherche qui lui donne son substrat : une observation prolongée du jeu politique local à Nanterre, des élections et des échanges entre élus et militants associatifs dans les Hauts-de-Seine entre 2005 et 2013, et l'immersion dans différentes associations de quartier. Le dépouillement des archives municipales, associatives, départementales et de mouvements historiques de défense des étrangers a en outre élargi l'analyse du très contemporain au retour sur la naissance des luttes autour des étrangers au sein du communisme municipal, de la guerre d'Algérie à aujourd'hui. Cette historicité et l'enracinement de la question immigrée à Nanterre facilitent le dépassement des « débats d'immigration » focalisés sur l'actualité ou des épisodes coloniaux hautement dramatiques, enfin sur certains microcosmes militants parisiens, déjà étudiés ${ }^{7}$. Car, si l'ascension des usages du passé colonial tient à la nationalisation des débats autour de la guerre d'Algérie et du procès de Maurice Papon à la fin des années 1990, et si le pic de projets régionaux sur la mémoire suit dans la période comprise entre 2003 et $2006^{8}$, l'importation de ces enjeux reste loin d'être mécanique. En matière de patrimonialisation, le national conserve une forte coloration locale : l'articulation à «l'identité » et au territoire sont essentielles. Seuls peuvent en rendre compte les échanges entre élus, habitants et militants associatifs ou de quartier, les discussions incessantes, officielles ou officieuses, sur les marchés, aux réunions publiques, électorales, aux cérémonies, soirées sportives, fêtes locales ${ }^{9}$. La reprise comme enjeu militant et l'at-

3. Retière J.-N., «Autour de l'autochtonie. Réflexions sur la notion de capital social populaire », Politix, n63, 2003, pp. 121-143.

4. Janvier L., « Du nouveau sous les ponts », La Mer à boire, Paris, Gallimard, 1987.

5. Laurens S., Une politisation feutrée : les hauts fonctionnaires et l'immigration en France, 19621981, Paris, Belin, 2009.

6. Noiriel G., À quoi sert l'identité nationale ?, Marseille, Agone, 2007.

7. Bertrand R., Mémoires d'empire. La controverse autour du "fait colonial », Bellecombe-enBauges, Broissieux/Croquant, 2006.

8. Collet V., Du bidonville à la cité. Les trois âges des luttes pro-immigrés : une sociobistoire à Nanterre (1957-2011), Thèse pour le doctorat de science politique, dir. Collovald A., Université de Nanterre, 2013, pp. 533-534.

9. Rougier C., «Usages politiques et appropriation "populaire” d'une tradition "réinventée". La Fête des Ponts à Limoges », Politix, vol. 23, n 92, 2010, pp. 125-143. 
trait municipal pour tel événement ou tel personnage historiques dépendent de ces échanges, de l'obligation de composer avec un milieu associatif très actif et d'une potentielle appropriation habitante des figures adoptées - locales, régionales, nationales voire internationales. La fabrique de la patrimonialisation s'éclairera ici d'une séquence - comprise entre 2003 et 2013 -, de lieux l'ancienne boucle Nord-Ouest de la Seine soit une partie des Hauts-de-Seine et du Val D'Oise - et d'une observation plus fine en forme d'exception Nanterre.

\section{À la recherche du temps perdu. Quand Nanterre ne veut plus oublier}

Si Nanterre fait figure d'exception, c'est à plusieurs titres au début du nouveau millénaire : pour son communisme municipal (depuis 1935), l'ampleur de ses cités HLM 10 et de sa population immigrée dans les très riches Hauts-de-Seine, pour sa rénovation urbaine à marche forcée avec l'extension rapide de la Défense sur la commune. Les célèbres bidonvilles algériens de Nanterre semblent loin. Cinquante ans plus tôt, ils abritaient jusqu'à près d'un tiers de la population communale, soit près de 20000 personnes. Lieuxsymbole du massacre des Algériens à Paris, ils représentaient aussi l'exceptionnel silence autour de l'événement. Car, si la brochure distribuée à chaque Nanterrien en 2011 fait du 17 octobre 1961 un symbole local, dix ans plus tôt, aucune cérémonie ne l'évoquait. De quel symbole le 17 octobre est-il donc le nom ? Avant tout, semble-t-il, d'un changement d'époque, où l'officiel «Nanterre ne veut pas oublier» efface qu'il s'agit en fait de ne plus oublier ce qui a longtemps fait problème ${ }^{11}$.

\section{Du 17 octobre à l'institutionnalisation municipale de la mémoire de l'immigration}

1991 : Pont de Bezons. La plaque commémorative de I'association «Au Nom de la mémoire » est descellée par le Préfet. Les « anciens de l'immigration " présents depuis des années sont rejoints par le groupe de Colombes «Action Citoyenne » dont plusieurs militants ont grandi dans les bidonvilles de Nanterre.

1999 : Une pétition « contre l'oubli » réunit des milliers de signatures, dont celle de la députée-maire de Nanterre Jacqueline Fraysse (PCF).

2001 : Paris. Le maire Bertrand Delanoë (PS) dévoile une plaque au Pont Saint-Michel sur la « sanglante répression de la manifestation pacifique ", ignorant la responsabilité policière et le couvre-feu racial. Colombes. La majorité municipale passe à droite ; I'opposition de gauche, les «Motivé-e-s » et le maire honoraire (PCF) rallient les commémorations au Pont de Bezons.

2002 : Nanterre. Après la tuerie au conseil municipal 12, les élections

10. Entre 2001 et 2011, stables en donnée brute, ils passent cependant de $55 \%$ à $48 \%$ du logement dans la ville.

11. Noiriel G., État, nation et immigration. Vers une histoire du pouvoir, Paris, Belin, 2001, p. 337. 
législatives consacrent une courte victoire à gauche sur la circonscription Suresnes-Nanterre.

2003 : «Année de l'Algérie », un colloque en hommage à Abdelmalek Sayad est suivi de la première commémoration officielle du massacre. La députée-maire pose une plaque similaire à la parisienne, face à la préfecture.

2004 : Création de l'association « Les Oranges 13 ».

2006 : Le nouveau maire Patrick Jarry reprend la proposition des Oranges pour un collège " Abdelmalek Sayad » en adressant un courrier au président du conseil général, Nicolas Sarkozy.

2007 : Commémoration du 17 octobre : le maire annonce qu'un bâtiment public «Abdelmalek Sayad » verra le jour ainsi qu'une rue « 17 octobre 1961 ». Clichy-la-Garenne (PS). Le maire fait poser une stèle pour le massacre au Pont de Clichy ; "Moh Clichy », ancien responsable FLN, est fait citoyen d'honneur.

2008 : Asnières-sur-Seine (PS), Colombes (PS, PCF, Verts). Le changement de majorité conduit les élus à commémorer le massacre. Nanterre. La liste plurielle emmenée par Patrick Jarry est réélue au premier tour.

2011 : Bagneux (majorité plurielle, PCF). La mairie fait poser une stèle pour les victimes. Malakoff (PCF). La mairie lance un appel à témoignages et au « devoir de mémoire » dans le bulletin municipal.

16 octobre : Nanterre. Le maire dévoile le boulevard " 17 octobre 1961 ». Colombes. La plaque commémorative, dévoilée en 2008, ne peut toujours pas être posée sur et sous le Pont de Bezons (refus préfectoral et du conseil général).

17 octobre: Pont de Clichy. François Hollande lance sa campagne en se rendant à la commémoration auprès du maire d'Asnières (rejoint par celui de Clichy). Esplanade de la Défense. Les maires d'Argenteuil, Asnières, Colombes, Nanterre, Gennevilliers et Clichy commémorent l'événement et se rendent au Pont de Neuilly malgré l'interdiction. Gennevilliers, Blanc-Mesnil. Inauguration d'une place 17 octobre 1961

2012 : Les maires (PS) d'Argenteuil, Asnières, Clichy-la-Garenne et Colombes appellent les autorités de l'État à la reconnaissance du massacre. L'Elysée I'officialise par communiqué : la responsabilité policière, le couvre-feu racial et le décompte des victimes restent absents.

2013 : Nanterre. Le journal municipal fait sa Une sur l'inauguration du groupe scolaire Abdelmalek Sayad. Peu après les « vingt ans de la mort d'Abdennbi Guémiah", le conseil municipal adopte le principe de deux rues au nom du lycéen de Nanterre et de la cité de transit où il résidait.

2014 : La municipalité inaugure le boulevard Abdennbi Guémiah en juin. À quelques centaines de mètres, un nouveau bidonville est expulsé un mois plus tard.

12. Dans la nuit du 26 au 27 mars 2002, après être passé par plusieurs partis et associations de gauche, Richard Durn tue huit élus au conseil municipal et en blesse dix-neuf dont quatorze grièvement. Neuf conseillers sont décorés de la légion d'honneur comme les huit conseillers tués, à titre posthume. Peu évoquée, la tuerie atténue certainement les conflits politiques au conseil municipal. En mars 2012, huit rues sur l'Axe Seine-Arche prennent le nom de chaque conseiller disparu.

13. En référence à la pièce d'Aziz Chouaki jouée au théâtre des Amandiers quelques temps auparavant par le président de l'association. 
Car très vite, le 17 octobre se trouve alors enrôlé dans la question de la " résorption », c'est-à-dire dans la bataille municipale contre l'État afin d'obtenir des garanties sur la destruction des bidonvilles et le relogement des occupants ${ }^{14}$. Et il disparaît du débat jusqu'aux années 1990. Sa réapparition signifie en parallèle un changement de configuration, au-delà du retentissant procès de Maurice Papon en mai 1998 et du témoignage de Jean-Luc Einaudi ${ }^{15}$, « événement dans l'événement » qui fait resurgir médiatiquement le massacre des Algériens.

Le retour du souvenir public n'est, en effet, pas exempt de singulières sélections. La violence exceptionnelle où les drames y sont centraux, les grandes figures de l'immigration et de la colonisation aussi. La violence brute du nouveau « devoir de mémoire 16 », à l'image du 17 octobre 1961, met souvent à l'encan le temps long : celui des stigmatisations, des socialisations séparées à l'usine, du fossé creusé entre bidonvilles et cité ouvrière ordinaire. Or, cette longue séparation s'accommode difficilement de l'évaporation de tout ou partie des responsabilités locales, municipales, communistes, de la « gauche » en général. L'exposition de dommages extraordinaires accompagne la nouvelle ère d'usages du passé : l'événement, l'exception et les victimes priment l'ordinaire, le quotidien et l'organisation politique de l'immigration, mais aussi les soutiens de la première heure, souvent d'extrême gauche et/ou chrétiens. Est en général oublié, de même, le « déplacement du problème » que constitue alors " le remplacement des bidonvilles par les cités de transit ${ }^{17}$ ", manière pour la municipalité communiste d'en extraire les occupants de sa gestion au profit du département et de bailleurs spécialisés comme la Sonacotra et la Cetrafa ${ }^{18}$.

Exceptionnel et national, le massacre du 17 octobre semble ainsi bien seul (à l'exception de figures des indépendances) à rallier au-delà du local une histoire de l'immigration sans représentants. À Nanterre, il permet aussi à la municipalité de rallier la lutte associative et la mémoire des habitants sans se dédire : la dénonciation des responsabilités d'État de l'une s'associant à la volonté de reconnaissance des autres. Le passé resurgit ainsi selon les stratégies locales et souvent après un changement municipal : il est politisé par les élus de gauche plurielle passés dans l'opposition à Colombes après 2001, ou une fois ces derniers installés à la tête de la municipalité comme à Asnières et à Argenteuil dix ans plus tard.

14. De Barros F., L'État au prisme des municipalités. Une comparaison historique des catégorisations des étrangers en France (1919-1984), Thèse pour le doctorat de science politique, dir. Offerlé M., Université Paris 1, 2004, pp. 254-256 ; pp. 361-366.

15. Riceputi F., La bataille d'Einaudi. Comment la mémoire du 17 octobre 1961 revint à la République, Paris, Le Passager clandestin, 2015.

16. Gensburger S., Lavabre M.-C., «Entre “devoir de mémoire” et “abus de mémoire” : la sociologie de la mémoire comme tierce position », in Müller B. (dir.), L’histoire entre mémoire et épistémologie. Autour de Paul Ricoeur, Lausanne, Payot, 2005, pp. 75-96. 
La mise en mémoire des rapports entre " la gauche " et les populations immigrées

L'aggiornamento ne va pourtant pas tel un long fleuve tranquille et simplement électoral. La conjoncture nationale, qui fait réémerger ce passé colonial, n'est opportune que parce qu'elle est saisie et mise en forme par des militants de la cause de la mémoire. Autrement dit, si la conjoncture est bonne, la manière de « dire le problème » reste essentielle : l'adhésion des décideurs en dépend. À Nanterre, la multiplication d'événements, de mobilisations associatives, les sollicitations permanentes aux débats, cérémonies et lieux où se croisent élus et militants façonnent cette ascension entre 2004 et 2009, dans le sillon des élections municipales de mars 2008. À force de répétition et de partage de vues, les passés de l'immigration et l'enjeu de la « diversité » prennent un ton nanterrien et s'officialisent dans des actes publics. Si le maire et ses adjoints l'expriment ouvertement après 2005, c'est qu'ils sont plus convaincus de la justesse de l'enjeu et des mots pour le dire mais aussi en raison de la multiplication des initiatives et des demandes en ce sens dans des associations variées, de l'aide à la scolarité pour les plus jeunes aux centres sociaux et associations militantes.

C'est aussi que, dès les années 2000, les controverses sur l'histoire mouvementée de la « gauche » avec les populations immigrées deviennent très visibles à Nanterre. La sortie en 2001 du livre de Monique Hervo, établie entre 1959 et 1962 au bidonville de « la Folie », rappelle le très dur quotidien du bidonville, le silence municipal et du voisinage sur le massacre et en général sur le sort des occupants de baraques. La commémoration parisienne la même année conduit plusieurs associations de Nanterre à revendiquer une cérémonie locale derrière le collectif « 17 octobre contre l'oubli ». Et en 2003, la déclinaison locale de l' " année de l'Algérie » accélère cette ascension publique. Les représentations artistiques, théâtrales et les conférences s'achèvent par la pose d'une plaque en mémoire du 17 octobre 1961 au pied de la préfecture et la préparation d'un ouvrage sur les Mémoires algériennes aux éditions Syllepse soutenu par un des adjoints au maire. Au lieu d'y mettre fin, l'ouverture municipale élargit les revendications associatives et débouche en 2004 sur la création de la première association focalisée sur une « mémoire positive de l'immigration » - les Oranges - et présidée par un ancien des bidonvilles de Sartrouville, M'hamed Kaki. Entre temps, le documentaire Nanterre, une mémoire en miroir, de Cheikh Djemaï, ancien des bidonvilles et de la cité de transit André Doucet à Nanterre, est projeté au cinéma municipal au début de l'année 2004, et donne une nouvelle centralité à la mémoire du massacre dans la ville.

17. Cohen M., «Bidonvilles de Nanterre : une destruction en trompe-l'œil », Plein droit, $\mathrm{n}^{\circ} 88$, 2011, pp. 32-36.

18. Entretien avec Bruno et Elisabeth Ehrmann, Paris, 14 novembre 2009. 
Ce moment de (re)politisation du passé colonial et de l'immigration prend bien un tour particulier après 2004. Le département est dirigé par Nicolas Sarkozy, dont les discours incessants sur la sécurité et l'immigration sont associés localement à l'emblème caricatural qu'il représente d'un département à deux vitesses en tant que maire de Neuilly-sur-Seine. À Nanterre, l'équipe municipale est alors en profond renouvellement après la tuerie de 2002 et la disparition d'une partie du conseil municipal. La députée-maire Jacqueline Fraysse organise peu après la passation de pouvoir au profit de Patrick Jarry. Le conflit bientôt ouvert entre ce dernier et la section communiste, notamment lors des élections municipales de mars 2008, s'achève finalement par la sortie des principaux membres du bureau municipal du giron communiste en 2010.

Entretemps, un événement national a fait changer le jeu d'échelle et contribué à l'ouverture de la municipalité aux revendications pro-immigrés ${ }^{19}$ : les «émeutes » de 2005. Leur importation locale, comme lors du débat organisé par les Oranges un an après les faits, conduit à multiplier les interpellations militantes et associatives sur l'intégration politique des enfants d'immigrés et la représentativité du conseil municipal. Les nominations de Rachida Dati, Rama Yade et Fadela Amara au gouvernement en 2007 accentuent le virage où passés colonial et immigré se croisent et intègrent les controverses sur la place des enfants d'immigrés en France et en politique. À Nanterre, les échanges au sein du microcosme politique local tournent alors quotidiennement autour de ces questions. Le parachutage d'un conseiller de Nicolas Sarkozy, Rachid Kaci, raffermit encore la tendance. Investi tête de liste UMP aux élections de mars 2008, il doit « troubler » le jeu nanterrien et rallier un « hypothétique vote musulman » tout en défendant une ligne «ultra-laiqque » et « anti-communautariste ». L'échec patent de l'opération renforce a contrario ceux qui s'en étaient saisis mais à gauche.

Pour une fraction des militants associatifs proches du jeu partisan, comme le président des Oranges, le passé est ainsi devenu (aussi) une ressource pour s'attirer l'écoute des professionnels politiques et face à des militants associatifs souvent mieux implantés dans les quartiers ${ }^{20}$. Manière de s'imposer de façon détournée, la mémoire coloniale et immigrée réévalue du même coup l'enjeu de la représentation politique des héritiers de l'immigration. Passé et présent s’y trouvent déclinés en des termes qui font écho au besoin des équipes municipales de regagner en représentativité. L'affichage d'une « diversité à la nanterrienne » en mars 2008, avec plus d'un tiers de la liste " gauche rassemblée » consacrée à la « diversité », en est une conséquence directe, que l'on retrouve alors en des termes similaires dans bien d'autres villes de banlieue ${ }^{21}$.

19. Mathieu L., «L'espace des mouvements sociaux », Politix, n77, 2007, pp. 131-153.

20. Collet V., « De la ressource intellectuelle à la cause politique. Les différentes vies d’Abdelmalek Sayad », Politix, n94, 2011, pp. 155-178. 
L'action militante est bien déterminante à Nanterre. La reprise des thèmes par l'équipe municipale semble inversement proportionnelle au poids des événements eux-mêmes : les émeutes passent presque inaperçues à Nanterre, les commémorations du 17 octobre y débutent tardivement, Abdelmalek Sayad y est inconnu publiquement avant 2003. L'enjeu est donc ailleurs, logé dans le signal adressé aux associations de quartier, au pôle militant pro-immigrés actif et majoritairement tenu par des enfants d'immigrés de la classe moyenne intellectuelle toujours liés à un électorat populaire profondément renouvelé dans les cités.

\section{Une définition localisée du colonial dans la refonte générale du rapport au "populaire »}

La mémoire du passé colonial et de l'immigration trouve dans cette configuration les clés de sa victoire contre l'oubli. Mais la victoire locale tient aussi, peut-être avant tout, à la métonymie opérée entre l'immigré et le populaire : derrière la patrimonialisation de "l'immigration ", l'adresse se fait au populaire. C'est bien dans ces allées et venues, de l'immigré au populaire, de l'associatif au municipal, que se construit la fusion entre passé immigré et patrimoine de Nanterre ${ }^{22}$. La nécessité de rénover la représentation politique et de s'adresser aux cités de Nanterre trouve dans le passé un relais symbolique et une ressource peu coûteuse pour l'exprimer. En pleine restriction budgétaire, le gouvernement des morts offre en effet, à coût quasi-constant, reconnaissance et affichage d'un volontarisme intact à l'égard des cités. L'aggiornamento politique s'en trouve précipité avec l'arrivée d'un grand nombre d'héritiers de l'immigration au conseil après 2008.

Le temps paraît ainsi révolu des grands ensembles passés de «l'emblème au problème » des banlieues ${ }^{23}$, qui a longtemps reconduit le malentendu entre politisation communiste et vie quotidienne des enfants d'immigrés dans les cités. Née de la séparation dans les années 1960 et marquée par la guerre d'Algérie, la tension culminait vingt ans plus tard autour du relogement des habitants des cités de transit, avec la plus longue lutte de jeunes «immigrés » de Nanterre, de novembre 1982 jusqu'au printemps 1985, suite à la mort d'Abdennbi Guémiah. La double revendication - pour la condamnation du meurtrier et pour le relogement des familles de la cité de transit Gutenberg où vivait le jeune lycéen - objective alors la séparation sociale, locative, professionnelle et quotidienne dans la ville communiste.

21. Masclet O., Sociologie de la diversité et des discriminations, Paris, Armand Colin, 2012.

22. Collet V., Du bidonville..., op. cit., pp. 692-695.

23. Tissot S., «De l'emblème au problème. Histoire des grands ensembles dans une ville communiste ", Annales de la recherche urbaine, n93, 2000, pp. 123-129. 
Or, une fois le logement exceptionnel détruit, la tension s'estompe lentement. D'abord avec le remplacement du maire Yves Saudmont par la jeune cardiologue Jacqueline Fraysse au milieu des années 1980. Les signes du basculement se lisent clairement dans le journal municipal, le Nanterre Info : l'attention croissante à la «culture immigrée ", aux spectacles et talents de Nanterriens venus d'ailleurs, supplante au milieu des années 1980 l'invisibilité antérieure et concurrence les thèmes jusque-là omniprésents de l'insécurité et la délinquance. Le sillon de la Marche pour l'égalité des droits et contre le racisme de l'automne 1983 se répercute localement, popularise une « culture beur " plus soluble politiquement que les conflits ouverts par les mobilisations pour « en finir avec le transit ». En ce sens, la déradicalisation des actions militantes des enfants d'immigrés après les marches de 1983 à 1986 accentue la tendance, de même que l'effacement de l'extrême gauche et des chrétiens sociaux qui portaient ces revendications. Mais c'est surtout l'explosion associative qui change la donne. La lente structuration d'associations de quartier et la vague de création au début des années 1990 se retrouvent à maturation au début des années 2000. Au moment même où les jeunes militants communistes des années 1980, qui dénonçaient les «vagues d'immigration » ou le trafic de drogue au Petit Nanterre, arrivent à la tête de la municipalité, et sont désormais les premiers à regretter le repli ouvrier et jugent criant le besoin d'ouverture sur les mouvements citoyens et associatifs. Certaines associations vont ainsi former l'ossature des sept centres sociaux et culturels mis en place avec la ville dont le statut associatif (pour six d'entre eux) affiche une grande autonomie de fonctionnement. Le rapprochement entre notoriétés de quartier et associations militantes fait le reste : l'association devient pour la municipalité un lieu privilégié mais plus indirect de recrutement de candidats au moment des élections, à mesure que pouvoir municipal et section communiste se disjoignent. La lente érosion électorale et militante du PCF, sa disparition de plusieurs quartiers comme l'affaissement des sociabilités communistes hors du noyau filial à la tête de la municipalité imposent une "rénovation politique ", qui s'accélère lors du second mandat de Jacqueline Fraysse. D’autant que certains des leaders associatifs sont aussi les premiers, dès 1995, à pointer le décalage entre élus et habitants, et qu'ils concurrencent désormais politiquement ceux qu'ils ont durablement remplacés dans les cités.

L'ouverture aux « initiatives citoyennes » sous Patrick Jarry suit cette dévaluation brutale de la marque partisane au milieu des années 2000 : effectifs en chute libre, arrêt de la parution du journal de la section, L'Eveil, réduction des activités de la section à la portion congrue avec un seul demi-permanent après 2007. La section PCF ne fait plus le poids face aux 2500 employés communaux. Mais le processus renforce du même coup l'incertitude sur les échéances électorales. L'élargissement des tractations et du casting en vue des élections municipales de 2008 en prend le relais, comme l'intérêt pour le passé et les controverses coloniales, témoin de l'ascension brutale des hauts diplômés au bureau municipal, comme dans tout l'appareil communiste ${ }^{24}$. 
Le triptyque parti-section syndicale-satellites associatifs (comités de quartier, de locataires, Secours populaire) a fait long feu. Il s'est dévitalisé au profit de structures plus indépendantes et sa succession est loin d'être garantie ${ }^{25}$. Les militants qui restent ou qui en ont pris le relais sont souvent moins directement monnayables sur le plan électif, issus d'un monde associatif plus à distance, qui doit composer avec ses adhérents et les risques d'illégitimité ou de tensions lors du passage en politique. La gestion des agents communaux ne peut à elle seule suppléer cette perte d'adhérence sous peine de s'attirer les dénonciations d'une instrumentalisation contraire à l'obligation de «neutralité administrative ». La crise de la section avec le noyau dirigeant accélère en conséquence le virage « citoyen et associatif » qui peut, contrairement à certaines autres communes populaires comme en Seine-Saint-Denis, s'appuyer sur un dynamisme économique important. Le soutien aux associations, la rénovation des quartiers, le co-financement des centres sociaux et culturels qui s'ensuit pèse nettement dans les conflits, plus feutrés, avec une municipalité qui pourvoie à l'essentiel des ressources associatives, financières, en prêts de salle, en aide à l'impression et à la communication.

L'apogée associative et la forte mobilisation des enfants d'immigrés dans les associations et les services de la ville travaillent à l'évidence la réception par les dirigeants politiques de l'enjeu mémoriel ou de la rénovation représentative. Le gouvernement du passé de l'après 2005 marque cependant une césure : la représentation et les mémoires de l'immigration sont passées d'enjeux « minoritaires» ou cantonnées à de simples souvenirs d'habitants à des enjeux publics prioritaires à défendre et à valoriser politiquement.

S'ils trouvent un point d'appui particulier à Nanterre, c'est en s'inscrivant dans le mouvement plus large des communes de banlieue populaire, hier encore ouvrière et communiste ou socialiste. Les transformations économiques, urbaines et politiques brutales y ont longtemps reconduit la perception d'histoires coloniales conflictuelles risquant de "déstabiliser » plus encore une classe ouvrière déjà bouleversée par la désindustrialisation et les crises internes au mouvement ouvrier. À l'inverse, une fois transformées en levier de reconnaissance, les mémoires, cultures et représentations de l'immigration se renforcent de la mise à distance du communisme comme horizon politique et comme héritage. Les groupes mobilisés autour de ces mémoires y trouvent alors autant d'opportunités d'imposer leurs passés aux décideurs locaux.

24. Pudal B., Un monde défait. Les communistes français de 1956 à nos jours, Bellecombe-enBauges, Broissieux/Croquant, 2009, pp. 176-182.

25. Mischi J., «Pour une histoire sociale du déclin du parti communiste », in Haegel F. (dir.), Partis politiques et système partisan en France, Paris, Presses de Sciences Po, 2007, p. 84. 


\section{Abdelmalek Sayad vs Abdennbi Guemiah : l'intellectuel et le bidonville, le jeune de Nanterre et le transit}

À compter du milieu des années 2000, le 17 octobre 1961 n'est plus seul au panthéon revendiqué de l'immigration. À sa suite, plusieurs noms et événements émergent. Leurs usages et appropriations sont plus divers qu'en réelle concurrence, a fortiori qu'en guerre 26. À Nanterre, la multiplication débute avec la lutte pour un collège au nom du sociologue Abdelmalek Sayad, qui entraine avec elle celle pour une rue au nom d'Abdennbi Guémiah. La figure nationale et intellectuelle d'un côté, le jeune de la cité de Nanterre de l'autre, expriment ces identifications différentes de nouveaux notables et militants de l'immigration. Les deux luttes de mémoire sont lancées presque au même moment et par des acteurs proches, candidats sinon depuis membres du conseil municipal. Et elles prennent une ampleur qui, après 2008, tend à en faire un enjeu local de premier plan. Ne plus mécontenter les uns, c'est reconnaître le passé des autres. La nécessité de l'ouverture politique devient cumulative en matière de patrimonialisation.

La transformation d'Abdelmalek Sayad en enjeu de mémoire politique à Nanterre n'a pourtant rien d'évident. Trois phénomènes s'y imbriquent étroitement : la conjoncture de sortie du passé colonial et sa connexion aux débats d'immigration; la redécouverte de Sayad, au-delà des cadres locaux de la mémoire immigrée, dans l'espace scientifique et militant au fil des (ré)éditions d'ouvrages posthumes; l'appropriation locale du sociologue grâce au travail de passeurs militants comme le président des Oranges. La trajectoire de M'hamed Kaki, des bidonvilles de la région aux universités, les liens construits dans celles-ci avec certains des chercheurs qui rééditent Sayad, font bifurquer aussi sa carrière militante : les références savantes deviennent pour lui une ressource dans les banlieues qu'il arpente, de Sartrouville à Mitry-Mory, de Gennevilliers à Nanterre. Contrairement aux postes politiques qui restent souvent fermés, les armes scientifiques se révèlent très efficaces pour toucher les élus, à défaut de subvertir réellement leur jeu.

Un premier pas est franchi avec le colloque en hommage à Sayad en 2003. Mais c'est aussi l'échec d'une proposition parallèle pour une station «17 octobre 1961 » sur la ligne 13 du métro, à Asnières-Gennevilliers ${ }^{27}$, qui recentre la lutte des Oranges sur le futur collège de la commune et sur Abdelmalek Sayad, d'octobre 2005 à septembre 2007. Deux séquences ponctuent la mobilisation. La première, jusqu'en avril 2007, est locale et suit un rythme irrégulier. La pétition mise en ligne par l'association recueille plusieurs milliers de signa-

26. Blanchard P., Veyrat-Masson I. (dir.), Les guerres de mémoire. La France et son histoire, Paris, Seuil, 2008.

27. Le président des Oranges y travaillait au service municipal de la jeunesse avant de déménager à Nanterre en 1999. 
taires d'horizons très divers, dont de nombreux universitaires. Mais, sans opposition déclarée, la lutte reste peu soutenue, visibilisée pour l'essentiel par une dizaine d'articles de presse et quelques interventions radiophoniques jusqu'à la fin 2006. La tentative avortée d'un second colloque en hommage à Sayad impose alors de relancer la lutte.

Le 24 mai 2007, l'inauguration symbolique du collège lance une nouvelle mobilisation de papier 28 : plusieurs articles de presse, certains nationaux, et des télévisions d'information en continu entrent dans le jeu. Les destinataires de la proposition sont tenus de se prononcer, à commencer par les représentants du conseil général et sa vice-présidente, Isabelle Balkany. Certains élus et militants de Nanterre interviennent aussi. La nouvelle phase rend ainsi visible la contre-mobilisation. Jusque-là, le silence du conseil général et ses contacts avec des membres du futur collège ou encore ceux plus souterrains des élus de Nanterre avec des acteurs associatifs plus rétifs à la proposition, imprimaient un « faux-rythme » à la mobilisation. La médiatisation oblige à rompre le silence mais elle transforme vite la question en polémique entre le " maire communiste » et le « département de N. Sarkozy ", et fait passer au second plan militants associatifs, soutiens intellectuels et fond de l'affaire. Les médias offrent surtout une occasion inattendue à l'équipe pressentie pour le collège d'exprimer ses craintes d'une «stigmatisation » de l'établissement par son association à un nom maghrébin. Éviter toute polémique politique et son « intrusion dans le sanctuaire scolaire » rend légitime un refus jusque-là indicible.

Au final, le nom retenu est celui de « République », rue sur laquelle se tient l'édifice. Les hésitations de l'équipe enseignante, reprises par le conseil général, déterminent le registre adopté pour rendre public le refus antérieur. «Dépolitiser » la question devient le mot d'ordre soutenant une peur profonde des enseignants et de certains associatifs du quartier. Pour les premiers, la peur tient à la croyance en une fuite (supplémentaire) d'élèves de milieux plus favorisés en raison de la « réputation » du collège, et l'homogénéisation d'un recrutement anticipé comme provenant essentiellement du Petit Nanterre, le plus pauvre et le plus «immigré » des quartiers de la ville. Pour les seconds, engagés dans l'accompagnement scolaire dans ce quartier, l'opposition est plus nuancée et associée à l'idée de porter la revendication ailleurs, afin d'éviter la stigmatisation. C'est le quartier qui est avant tout en jeu pour eux.

«Des enseignants du collège, dans un esprit de concorde républicaine, ont proposé le nom de République. Nous y voyons tout un symbole. [...] Notre République, c'est celle qui nous rassemble et

28. Champagne P., «La manifestation. La production de l'événement politique ", Actes de la recherche en sciences sociales, $\mathrm{n}^{\circ} 52-53,1984$, pp. 19-41. 
qui défend des valeurs comme celles de la laïcité et qui sait reconnâ̂tre la place importante de l'éducation. Ce collège portera haut les valeurs de la République qui sont aussi celles du respect et de la réussite de tous ${ }^{29}$ ".

La République reste «tout un symbole », comme le rappelle le principal du collège lors de l'inauguration officielle : mythe national naturalisé, il s'oppose au nom maghrébin qu'il renvoie instinctivement à son incompatibilité avec la réussite des plus défavorisés. La République s'enrichit d'une nouvelle facette de mise à l'écart du populaire. Masquée derrière l'opposition entre national et étranger, entre républicains et "communautaristes », elle se pare d'une lutte contre les stigmates sociaux, et pour la civilisation.

« Je crois à l'éducation, à la civilisation et à la vocation de l'État qui consiste à élever les citoyens, c'est-à-dire les rendre plus haut, pour leur apprendre à trouver en eux-mêmes les moyens de résoudre leurs problèmes. [...] Comme dans les familles, il arrive que le choix du nom de l'enfant engendre des polémiques. [...] La ville de Nanterre avait proposé de nommer le collège Ab... Abdelmalek Sayad et l'assemblée départementale n'a pas retenu cette proposition. [...] Je m'empresse de dire qu'il ne s'agit en aucun cas de contester la qualité des travaux d'Abdenmanek [sic] Sayad, sociologue élève de Bourdieu, remarqué pour son étude sur les populations immigrées de Nanterre. Mais ce choix était aux yeux des élus [...] porteur d'une trop forte connotation identitaire qui risquait d'enfermer ce collège dans une image communautariste, alors que nous souhaitons au contraire en faire un modèle de mixité sociale ! Et d'intégration! $30 »$.

Les travaux, la trajectoire, l'identité du sociologue, restent le ferment de cette altérité menant à l'échec. Antidote au "communautarisme », la République des professeurs et du conseil général ajourne la reconnaissance de l'immigration, associative et municipale, tout retournement du stigmate en emblème 31 étant alors voué à renforcer l'exclusion de ceux qui la combattent.

A contrario, l'intellectuel séduit avec la mobilisation certains acteurs municipaux et syndicaux qui vont en reprendre la lutte pour une des écoles de la commune. Sayad s'est en effet exporté au-delà du giron associatif et, si les médias ont importé le savant en se centrant avant tout sur lui comme enjeu de polémique, ils l'ont sorti des cercles militants et invité d'autres Nanterriens à se faire une opinion sur le sujet.

29. Discours de J.-P. Gratien, Principal du collège « République », 19 octobre 2007.

30. Discours de P. Devedjian, Président du conseil général des Hauts-de-Seine, 19 octobre 2007.

31. Goffman E., Stigmates. Les usages sociaux des handicaps, Paris, Minuit, 1977 [1963]. 
Souvent réduit à sa dimension locale (le collège se situant sur les lieux d'anciens bidonvilles) et à son statut de " grand intellectuel ", Abdelmalek Sayad est politisé, ajusté à tout ce qui peut influencer la décision politique et agréger des soutiens en laissant de côté l'autonomie de son œuvre ou ses travaux extérieurs à Nanterre. Les références des Oranges focalisées sur l'exemplarité algérienne sont associées aux scansions municipales, centrées sur la nanterrianité de Sayad, le maire ayant fait de l'enracinement local un levier essentiel de légitimation politique. Chacun fait de Sayad le représentant de sa cause, entérinant au final son importance comme symbole local. Les références à l'intellectuel et aux sciences sociales envahissent ainsi le discours des Oranges à mesure qu'elles pénètrent les arcanes municipaux. Car, en sortant Sayad de l'oubli et en le nanterrisant, les Oranges confortent leur monopole sur l'importation du sociologue et sur la question de la non-reconnaissance des « héritiers de l'immigration ", qui signifie souvent en pointillé la leur. La transitivité politique du sociologue fait le reste : la portée critique des textes de Sayad et son appel à une « existence politique de l'immigration » rejoignent le monopole d'identification qu'il détient auprès de ces militants d'origine maghrébine comme intellectuel maghrébin, algérien de surcroît, critique et contemporain. Sa réévaluation scientifique et politique, en se personnalisant, parachève la transformation de l'intellectuel en étendard militant et posthume. Elle connaît un épilogue heureux avec l'inauguration officielle du groupe scolaire Sayad en septembre 2013 par le maire de Nanterre, à nouveau en présence de Patrick Devedjian. La cérémonie concrétise l'accession (de longue haleine) de Sayad au panthéon local à la manière dont hier, les frères Fontanot, Nanterriens d'origine italienne, résistants et fusillés au Mont Valérien ou les membres du groupe Manouchian et de "l'affiche rouge », marquaient le présent communiste et la résistance comme identifiants de la Libération.

Mais l'aura nationale de Sayad et sa « mémoire » provoquent des réactions, y compris chez certains militants de quartier et « anciens » de Nanterre, au point de faire émerger dans son sillage une autre figure. Parfois opposée à cette "importation d'en haut ", celle, locale et populaire, d'Abdennbi Guémiah, émerge peu après le cinquantenaire. Abattu en 1982 par un voisin raciste, le jeune lycéen rappelle un habitat emblématique resté dans l'ombre des bidonvilles : la cité de transit.

De fait, le stigmate reste bien plus à vif, pour les familles concernées par cet habitat d'exception mais aussi pour les élus contemporains. Les tensions sur la question du « relogement » et de « l'éparpillement des immigrés » sont loin d'être oubliées. Et Abdennbi Guémiah force le trait puisqu'il résidait " cité Gutenberg », dite " cité blanche », là où émerge la plus radicale des luttes de résidents et celle aussi où la rupture entre « communistes » et « immigrés » a été la plus marquée. Or, la cité reste très «présente »: de nombreux anciens résidents habitent toujours Nanterre, notamment aux cités du Chemin de l'île 
et Pablo Picasso, et ils entretiennent souvent collectivement ce passé marquant.

Le mécanisme consistant à sortir le passé de ces mémoires habitantes et à en faire un enjeu public croise ici autant la conjoncture nationale que le changement municipal, avec l'arrivée au conseil d'anciens habitants de la cité. Entre octobre 2007 et juin 2013, entre l'officialisation par le maire de Nanterre d'un bâtiment public au nom d'Abdelmalek Sayad et le vote par le conseil municipal de deux rues - «cité blanche » et «Abdennbi Guémiah »-, les figures des indépendances, des Antilles, du massacre du 17 octobre se sont multipliées dans les rues de Nanterre. Le conseil municipal a été profondément renouvelé et, parmi les élus aux visages de cette " diversité à la nanterrienne », nombreux sont ceux qui reprennent à leur compte ces luttes mémorielles, certains les découvrant une fois arrivés au conseil. Ils attisent en retour le désir de Nanterriens de voir ce qu'ils considèrent être « leur histoire " s'inscrire au patrimoine local. Côté municipal, tous les signaux semblent en indiquer l'opportunité, c'est-à-dire la faisabilité : la baisse de conflictualité autour de ce passé du «transit » de même que l'intégration au conseil de plusieurs anciens de la cité semblent rendre l'épisode plus soluble dans l'institutionnalisation municipale.

En fait, très tôt, une première plaque en hommage au jeune lycéen a été apposée sur les grilles du collège André Doucet, où Abdennbi Guémiah étudiait quelques années avant sa mort. Mais, deux décennies plus tard, c'est la mobilisation pour Sayad qui fait ressortir les discussions autour d'une rue à sa mémoire. Plusieurs militants et habitants évoquent le « jeune de Nanterre qui a grandi et vécu là » par opposition à Abdelmalek Sayad et aux Oranges, dont les membres sont de récents Nanterriens, et dont certains habitants de longue date considèrent qu'ils politisent parfois des personnages et des passés étrangers à leur histoire.

Avant tout objet de discussions interpersonnelles, la revendication devient réellement publique avec la double commémoration qui se tient pour les trente ans de la mort d'Abdennbi en octobre 2012. La municipalité se trouve alors au milieu d'un jeu de revendications distinctes et pour partie concurrentes. L'une d'elle, "Abdennbi : trente ans déjà », est orchestrée par les anciens militants du relogement. Elle a lieu dans une des salles municipales du quartier du Chemin de l'Île, où sont exposés les clichés du quotidien et de la lutte dans la cité. Initiée par un des militants désormais élu au conseil municipal, Norredine Iznasni, ancien du Mouvement de l'immigration et des banlieues (MIB), elle est marquée à gauche et comme initiative municipale. Elle est finalement boudée par les organisateurs d'une seconde commémoration, tenue plus tôt le matin et où se rend, après de nombreuses discussions internes, une bonne partie des élus de Nanterre. La présence annoncée de 
nombreux membres de la famille et d'anciens de la cité habitant le quartier, détermine le revirement. La commémoration, relayée par plusieurs journaux, réunit au final près d'une centaine de personnes face au collège André Doucet.

Comme le rappelle un des militants universitaires de l'historique lutte des Gutenberg, Mogniss Abdallah, la séparation des commémorations rappelle celle qui existait dans la cité à l'époque ${ }^{32}$. Une fraction des jeunes, en grand surnombre dans la cité, plus proche du militantisme autonome et du milieu des bandes ${ }^{33}$, était alors très critique des organisations politiques et du PCF. D'autres familles et de nombreux jeunes, à l'inverse, étaient déjà plus proches d'associations cultuelles ou des institutions en place avec lesquels certains leaders négocieront des relogements individuels, des postes ici et là, pendant et surtout une fois la lutte terminée. Car cette fraction, plus rétive à la politisation du sort des habitants de la cité, se trouve alors comme effacée par le drame et la lutte pour la justice et le relogement. La mort d'Abdennbi met en suspens ces tensions préexistantes au profit du pôle militant qui coordonne alors le Comité Gutenberg. Mais elle refait surface trente ans plus tard autour du souvenir en mettant aux prises deux types d'engagement : l'un très politisé, hier à l'extrême gauche voire de façon " autonome ", parfois hors de Nanterre, qui s'est peu à peu rapproché d'une municipalité ayant fait son aggiornamento sur la question immigrée ; le second, plus proche d'un pôle de notoriété de quartier, à la frontière du sport, de l'engagement religieux et d'un « a-politisme » revendiqué par des représentants ayant vivoté entre listes électorales diverses et des emplois dans des municipalités de gauche et de droite de la fin des années 1980 à aujourd'hui, comme les frères Selmet. C'est peut-être cette hétérogénéité, et une commémoration scindée mais importante des deux côtés, qui accélère sa reprise comme enjeu de patrimoine par la ville, soucieuse d'éviter tout conflit avec cette clientèle ancienne et désormais historique de Nanterre.

L'épisode témoigne en tout cas d'un vrai changement : passé, identité et commémorations semblent être devenus pour partie un mode d'accession en politique et une modalité à part entière de lutte entre divers protagonistes. Norredine Iznasni, du premier pôle, marque le progressif raccrochage à la politique municipale en mars 2008, à partir du soutien du maire de Nanterre à la candidature José Bové aux présidentielles dont Norredine était un des porte-parole « de quartier », et du conflit avec la section communiste qui s'ensuivit. Quant à la réussite inattendue de la cérémonie du pôle plus «a-politique », lié à l'actif «blog de la cité blanche », elle se poursuit d'une série d'entretiens avec des personnalités de Nanterre et milite pour une rue au nom de la « cité blanche ». Elle consacre l'ascension de Mohammed Selmet sur les

32. Entretien informel avec Mogniss Abdallah, seconde commémoration pour « Abdennbi Guémiah », octobre 2012.

33. Mauger G., Les Bandes, le milieu et la bohème populaire. Etudes de sociologie de la déviance des jeunes des classes populaires (1975-2005), Paris, Belin, 2006. 
listes municipales aux élections du printemps 2014, occasionnant quelques conflits internes du fait de l'aspiration de revendications considérées comme citoyennes. Entre temps, la revendication s'est officialisée et même dédoublée : deux nouvelles avenues sur l'axe de l'Arche de la Défense à la Seine portent les noms de la «cité blanche » et d' « Abdennbi Guémiah », symbole de la satisfaction municipale de deux types de revendications mémorielles en partie clivées.

Le gouvernement de ces passés de l'immigration connaît pourtant des épilogues moins heureux, dont le boulevard Abdennbi Guémiah reste l'exemple. Relativement éloigné du jeu militant, le plus jeune frère de la victime, Hassan Guémiah, avocat et habitant du Chemin de l'île, s'est retiré peu avant l'inauguration de ses préparatifs, considérant que l'exposition à la mémoire de son frère éludait à nouveau les responsabilités municipales dans le drame et la gestion désastreuse des cités de transit. Invité à s'exprimer en juin 2013, devant un parterre d'élus perplexes, son discours pointe «l'application avant l'heure du programme du Front national par le PCF » et la présence «d'un nouveau bidonville, à quelques mètres d'ici ». Or, ce présent du bidonville, la municipalité l'occultait depuis des semaines. La diffusion d'un tract sur la question en plein rassemblement par plusieurs militants ajoute à la tension, le frère de la victime, dernier à prendre la parole et sous quelques huées, invitant les autorités à prendre leur " responsabilité pour qu'un nouveau drame ne se reproduise pas ». Il rejoindra, quelques jours plus tard, le collectif de soutien aux habitants, délogés à de nombreuses reprises, avec l'accord et le concours de la municipalité, de la préfecture et de l'aménageur de La Défense.

Un passé «immigré » qui, finalement, ne passe pas ${ }^{34}$. Ou pas si facilement. C'est l'un des sens de ce gouvernement des morts. Conviée et politisée pour dépasser les erreurs et les drames d'hier, la mémoire de l'immigration exprime autant une volonté politique de donner cohérence et identité à un présent social et urbain bouleversé de la ville populaire. À Nanterre, on affiche une réconciliation et un «vivre ensemble » au passé qui, de toute évidence, échappe au présent. L'ambivalence de cette institutionnalisation fulgurante de nouveaux passés n'est jamais aussi visible que lors de commémorations comme celle en hommage à Abdennbi Guémiah. Vandalisée depuis, la plaque à sa mémoire devait révéler le dépassement d'un passé qui se reproduit sous les yeux mêmes des commémorants, rivalisant d'ingéniosité pour se soustraire à la vue du nouveau bidonville et de l'ostracisme réitéré pour les quelques étrangers occupant les rares interstices persistants de Nanterre. La patrimonialisation a donc bien d'autres vertus que celle, affichée, «d'en finir avec ce passé ».

34. Conan E., Rousso H., Vichy, un passé qui ne passe pas, Paris, Fayard, 1994. 
Au-delà de l'ascension publique, l'inauguration de la rue Abdennbi Guémiah au même titre que l'intronisation locale de Sayad interrogent en effet les liens complexes entre passé patrimonialisé et présent "immigré », l'évolution des rapports à l'« immigré » enraciné et la persistance du regard sur les « étrangers » récemment arrivés. Une chose est sûre : la recherche de légitimation, forgée à l'extérieur du local et intellectualisée dans le cas de la lutte pour Sayad, a facilité l'ascension des passés coloniaux et «immigrés » en abaissant leur conflictualité auprès des élus, ouvrant la voie à l'intégration d'autres passés plus à vif comme celui du «transit ».

\section{Quand culture et passé « immigrés » effacent l'étranger : rénovation urbaine et gouvernement du passé}

La gouvernance locale du passé, comme lutte pour la représentation du monde, est une lutte avant tout symbolique ${ }^{35}$. Mais dire, c'est aussi faire : en cherchant à exprimer le renouvellement en cours, elle refonde le lexique municipal qui l'énonce. L'ascension d'une «diversité nanterrienne » et de « l'héritage populaire et immigré » suivent l'effacement de la question ouvrière et communiste.

La congruence entre la concurrence associative sur les passés de l'immigration et l'attention municipale croissante révèle ce qui se joue en termes de représentation et de représentativité : il s'agit d'une adresse directe à une population des cités enracinée sur le territoire et considérée comme une clientèle prioritaire. La situation contraste avec la dévitalisation du passé communiste et la disparition de ses conseillers au bureau municipal. La force des porteurs de la mémoire des uns suit une courbe inverse à celle des autres. Le groupe de référence d'hier, invisible et inaudible, prolonge dans la mémoire collective son effacement social au présent. Passé communiste et «immigré » suivent là leur chemin longtemps séparé. Et l'ascension du second signale une séquence générale de re-conversion des regards dans l'historique «banlieue rouge ». Les nouvelles vies nanterriennes d'Abdelmalek Sayad expriment bien un moment où des acteurs comme le maire de Nanterre, et sa lente sortie du giron communiste, posent un regard critique sur «le » Parti, rénovent le discours municipal en s'appuyant sur de nouveaux identifiants qui rompent avec la longue invisibilité politique des populations immigrées.

La réussite de l'entreprise de patrimonialisation locale se lit aussi dans les allées et venues de militants associatifs vers le conseil municipal et dans la circulation de discours sur " l'immigré et le populaire » au-delà des commémorations. Irriguant jusqu'aux campagnes électorales, l'aggiornamento sur le passé se fait toujours plus présent. Après 2008, les rues et bâtiments au nom de

35. Bourdieu P., «La représentation politique. Éléments pour une théorie du champ politique », Actes de la recherche en sciences sociales, $n^{\circ} 36 / 37,1981$, p. 8. 
l'immigration et des indépendances rejoignent celles en hommage aux disparus de la tuerie de 2002. Les morts s'y trouvent comme chargés d'endiguer le bouleversement présent - urbain, social et politique - avec la vertu supposée de redonner cohérence à l'histoire locale en s'appuyant sur ses groupes anciens et stables. Que le gouvernement des morts prenne ses quartiers sur l'Axe de La Défense n'est pas qu'un symbole. Opportunité pratique pour les nombreuses rues ou places qu'il ouvre aux patrimonialisations, l'Axe est aussi la contradiction la plus éclatante entre la revendication d'une continuité locale et la brutale transformation de la commune. Sur les vestiges du bidonville de La Folie devenus sièges d'entreprises bancaires et d'assurance, les morts de l'immigration, des massacres coloniaux et les victimes du conseil municipal se retrouvent chargés de sens, de soutenir le présent en énonçant le passé, de faire patrimoine là où, précisément, il a disparu ou n'existe pas encore. De fait, la résistance accompagne celle, plus radicale, d'autres morts : ceux, voisins, des cimetières de Puteaux et de Neuilly-sur-Seine, construit à Nanterre en 1886 pour décharger la commune voisine huppée et son premier cimetière saturé. Leur présence à l'entrée de l'Arche de la Défense contraint l'extension tout en hauteur du quartier d'affaires.

Structurer et ré-articuler le présent à un ou des patrimoines communs, redonner un continuum à des espaces à plusieurs vitesses, à des histoires à échelles multiples et à des groupes hétérogènes, y compris au sein du groupe « immigré » des cités, tel semble être l'ambitieux programme dévolu au passé. Il n'est évidemment pas le seul dans cette tentative de maintenir ou recoudre des histoires de plus en plus déliées. Certains quartiers d'habitat HLM de Nanterre, rénovés à marche accélérée, se retrouvent en effet physiquement et socialement méconnaissables en quelques années. Et le phénomène redouble à l'approche de l'Axe Seine-Arche et du quartier en chantier dit de "Nanterrela Défense ». Comme aux abords du stade de France et de la Plaine SaintDenis, les conflits générés par ces projets dépassent des communes relativement impuissantes à en maîtriser le développement. Le changement sociodémographique est majeur, l'embourgeoisement du parc privé très rapide, et l'arrivée de populations étrangères à l'histoire politique de la ville populaire massive. Nanterre passe ainsi de près de $80 \%$ d'ouvriers et d'employés au milieu des années 1970 à moins de 50\% au milieu des années 2000. Seule l'imposant parc social, moins mis en question que dans les villes alentours, freine le phénomène.

La forte tertiarisation de l'activité et le renouvellement de population n'en demeurent pas moins un dilemme pour la municipalité. En parallèle, le cœur politique nanterrien, ce foyer militant et associatif de quartier, reste arrimé à cet héritage immigré et populaire. Le passé de l'immigration exprime cette volonté de maintien voire de conservation d'une identité commune et fédératrice, adressé aux nouveaux arrivants mais aussi à cette clientèle des cités. Car, 
pour cette dernière, l'identité ouvrière et communiste n'est plus en mesure d'en assurer la continuité. Le passé «immigré » et le renouvellement municipal s'alimentent mutuellement pour ralentir la tendance et redonner un sens social aux mutations en cours, souvent visiblement effectuées au détriment des classes populaires. La rénovation représentative suit le même processus : reproduisant quasiment à l'identique le bureau dirigeant, elle s'adjoint symboliquement une fraction de la génération militante et intellectuelle « héritière de l'immigration » en forte ascension, mobilisée autour de son passé et qui a supplanté l'endocratie communiste et ouvrière d'hier dans les quartiers.

\section{Quand patrimonialiser, c'est faire exister}

Il faut bien replacer l'enjeu de ces patrimonialisations dans une configuration plus large : après plusieurs décennies de fractures occasionnées par les grands axes et les infrastructures étatiques, l'opposition de la ville " rouge » aux «meurtrissures de son territoire » se poursuit face aux nouveaux projets urbains. Convoquer l'histoire des massacres, des bidonvilles et de la colonisation, c'est prolonger la distinction avec le département et les villes alentours.

Confidentielles et tardives, les célébrations du 17 octobre à Nanterre passent ainsi en quelques années au rang de moteur du cinquantenaire. Le changement est budgétaire : l'événement mobilise les services techniques de la ville, la communication visuelle et papier est omniprésente, des chercheurs sont recrutés un an à l'avance pour organiser un colloque international ${ }^{36}$. Le public est d'ailleurs en forte croissance, hétérogène et plus jeune. Le changement est aussi politique : la contrainte associative est devenue engouement municipal. Le discours du maire lors de l'inauguration du boulevard du 17 octobre en témoigne. Après un long récit mêlant témoignages de Nanterriens et faits historiques, le maire politise l'événement en interpelant les autorités actuelles et en appelant à braver l'interdiction de rassemblement :

«Cinquante ans après, c'est par le silence ! Ou le mépris que les plus hautes autorités continuent à traiter ce drame. Que dire de certaines attitudes, telle celle du maire de Neuilly, qui prétend empêcher que l'on se rassemble demain près du pont de Neuilly, sur les lieux de la tragédie. N'en déplaise à monsieur Fromentin, nous serons demain nombreux à 17 h30 sur le pont de Neuilly (Applaudissements nourris)... Il est temps de faire entrer la date du 17 octobre 61 par la grande porte de notre histoire. Cette demande de reconnaissance officielle [...] portée par toute la gauche n'est ni la recherche d'une rédemption ni un acte de repentance. C'est une exigence de justice

36. Les historiennes Marie-Claude Blanc-Chaléard et Muriel Cohen travaillent à la préparation du colloque et à l'élaboration du programme de 8 et 16 pages (en couleur) déposé dans chaque boîte aux lettres de la ville. 
pour les victimes et leurs familles, de vérité et de morale pour nousmêmes et les générations à venir ».

Les centaines de manifestants ayant fait le déplacement le lendemain, au milieu des tours de la Défense, concluent avec succès l'opération. Le maire de Neuilly-sur-Seine n'y est pas étranger, après avoir déclaré :

«Par votre démarche, vous faites resurgir le souvenir d'une période extrêmement troublée durant laquelle notre pays était en guerre, générant des tensions et violences qui ont fait de nombreuses victimes parmi les deux pays belligérants. Après avoir évoqué votre initiative avec les associations d'anciens combattants, je regrette de devoir vous informer que je ne souhaite pas accueillir à Neuilly-surSeine la manifestation d'ampleur régionale que vous souhaitez y organiser, considérant que ma commune n'a jamais été associée à ce terrible événement ».

Les communiqués dénonçant la «falsification historique » des propos du maire de Neuilly y répondent immédiatement. Or, la ville-symbole de Neuilly-sur-Seine en redouble l'effet : le passé à vif ne passe définitivement pas, et semble même devenu plus conflictuel que certains enjeux économiques et sociaux. Relayée par des quotidiens nationaux, l'opposition du maire de Neuilly-sur-Seine contribue certainement à la réussite, incertaine, du rassemblement. Chevauchant des commémorations, peu annoncée dans les brochures locales et boudée par certaines associations, la cérémonie entre communes des Hauts-de-Seine et du Val d'Oise semblait mal amorcée. En politisant l'enjeu, le maire de Neuilly lui offre un contre-symbole fédérateur de la dénégation persistante des «institutions » et d'un mépris plus général (et de classe) pour les citoyens de banlieue. Elle ragaillardit l'événement et le rend très actuel, malgré son organisation malaisée un soir de semaine et en un lieu éloigné de plusieurs des communes associées.

Pourtant, malgré la coordination, les allées et venues de militants sur les marchés et leur contact avec certains journalistes, la marche des Hauts-deSeine restera peu visible. Le numéro spécial de L'Humanité sur le massacre lui préfère par exemple le défilé parisien et ses têtes d'affiche dans sa double page sur les commémorations ${ }^{37}$. La présence du candidat à l'élection présidentielle François Hollande à Asnières-sur-Seine retient l'attention télévisuelle, de même que la proposition de loi déposée au Sénat portant « reconnaissance de la responsabilité de la République française dans le massacre 38 ».

37. «Un crime d' État », L'Humanité, 17 octobre 2011.

38. Elle est déposée le 18 octobre 2011 par la sénatrice (groupe CRC) Nicole Borvo Cohen-Seat. 
Dans la longue marche contre l'oubli en banlieue et à « gauche », l'épisode aura pourtant marqué un pic inédit des rapports entre mouvements revendicatifs pro-immigrés et institutionnalisation municipale du souvenir. À Nanterre, il renforce les cadres de cette mémoire immigrée qui intègre dans la foulée à son panthéon de nouveaux drames et figures de l'immigration. Pour ce faire, le massacre est associé, comme Abdelmalek Sayad ou plus tard Abdennbi Guémiah, à la très ancienne opposition de la municipalité à l'État, au département et aux villes alentours. L'enrôlement de Neuilly-sur-Seine est là encore chargé de sens : les interminables luttes autour de l'aménagement de la Défense ont vu quelques temps plus tôt les élus de Neuilly, notamment Jean Sarkozy, tenter de prendre la tête de l'aménageur de la Défense et d'annexer une immense partie du territoire nanterrien. Le boulevard du 17 octobre n'est donc pas par hasard, au pied de la préfecture, du grand Axe Seine Arche et du conseil général.

\section{L’inauguration du boulevard du 17 octobre 1961, moment-clé des célébra- tions}

Son inauguration ponctue d'ailleurs avec force les événements qui s'enchainent lors de ce cinquantenaire. En pleine écriture de thèse, les commémorations deviennent d'ailleurs vite impossible à suivre tant les événements se chevauchent. Le retour au terrain intervient pourtant après la sollicitation d'une journaliste de L'Humanité pour un numéro spécial sur l'événement qui conduit à arpenter avec des militants les marchés et bars "algériens » où se retrouvent d'anciens manifestants, à suivre les célébrations à Colombes, Nanterre, La Défense et Paris, à collecter tracts, affiches et bulletins d'infos de la boucle nord (Bezons, Argenteuil, Colombes, Nanterre, Asnières et Clichy) et sud de la Seine (Malakoff, Montrouge). Du 14 au 17 octobre, le rythme est très soutenu. Après la projection du film de Yasmina Adi, Ici on noie les Algériens, qui fait salle comble à Nanterre et l'imposant colloque conclu le lendemain par un concert de la troupe Barbès-Café, l'inauguration du boulevard à Nanterre devient un moment phare. Le 16 octobre au matin, les discours associatifs et d'" officiels Algériens » s'enchaînent sur le parvis des Droits de l'Homme puis se déplacent sur la monumentale place Nelson Mandela. Des centaines de personnes sont présentes sous un ciel radieux. Le discours du maire Patrick Jarry clôt la cérémonie. Les visages sont empreints de recueillement, souvent émus face à la violence des faits racontés. Le maire dévoile la plaque du boulevard sous les applaudissements et les youyous, laissant place à la célébration musicale (et dansante). Plusieurs familles et « anciens » suivent les officiels, d'autres entament un défilé de photos avec le maire et les élus, beaucoup vont et viennent aux tentes abritant petits fours 
et auteurs (Didier Daeninckx, Olivier Le Cour Grandmaison). De grands panneaux explicatifs soutiennent l'étonnant décor : l'axe Seine Arche, en chantier et en arrière-plan, et l'immense préfecture enserrent le boulevard. Aux habituels élus et « anciens de l'immigration » s'est joint un important contingent de quadragénaires et de familles. De nombreux employés de la ville, presque tous enfants d'immigrés, en tee-shirt rouge et à l'imposante carrure, encadrent l'événement.

À gouverner par les morts et par le symbolique, le risque est pourtant grand, à terme, de fossiliser un passé commémoré jusqu'à ouvrir la voie à une célébration festive, en perdant de vue le processus qui lui a donné corps. Derrière les mots, c'est le lien politique dans les villes de banlieue que questionne ce gouvernement du passé. La politique de la mémoire interroge la transformation des groupes populaires, l'établissement de certains et la reproduction des conditions pour d'autres. L'inattendu retour des bidonvilles, de l'étranger, à Nanterre, en est un symbole paradigmatique. Il se trouve comme effacé par le passé institutionnalisé, les bidonvilles et les cités de transit d'hier, c'est-à-dire par l'immigré. L'équilibre entre passé et présent demeure donc fragile, sur une ligne de crête entre lutte pour la reconnaissance et le risque, en l'institutionnalisant, de la fixer au point d'empêcher d'en saisir l'actualité, ses différences et ses permanences, méconnaissables dès lors qu'elles s'appliquent à d'autres «types » de population catégorisées et mises à l'écart à l'identique, toutes configurations non égales par ailleurs, comme l'expriment certains anciens des bidonvilles ${ }^{39}$.

La politique publique de reconnaissance marque cependant une revalorisation certaine du patrimoine de l'immigration à travers son intégration locale qui actualise la critique des discours dominants sur l'immigration. Elle rappelle au passage que, même à propos de sa mémoire, l'immigration ne cesse d'être hétérogène, multiple : locale ou nationale, populaire ou intellectuelle, mobilisant des acteurs et des groupes distincts. Ce va-et-vient incessant du passé au présent et entre les groupes n'est jamais aussi visible que lorsqu'il émane de l'un des rares militants à l'origine des commémorations communes du cinquantenaire.

«Mesdames et messieurs, je ne vais pas vous parler du 17 octobre 1961 comme on a l'habitude de le faire. Depuis des années, des décennies, nous venions ici, au Pont de Bezons. Au tout début, c'était après 1973. Cette année-là, le sang avait beaucoup coulé. Le sang des Algériens. La nationalisation du pétrole a déclenché une nouvelle vague de haine raciste. Les Algériens, on les tirait comme des lapins. Attentat contre le consulat de Marseille. Au bout de l'an-

39. «Nous étions les Roms de l'époque », L'Humanité, 22 juillet 2014. 
née, près d'une centaine de morts. Nous venions au Pont de Bezons, avec le mouvement des travailleurs arabes. Il y avait H'med, il y avait Saïd, il y avait Mokhtar, et d'autres camarades... C'étaient les hommes de Radio soleil et de Sans Frontière. Ils étaient Palestiniens, Marocains, Tunisiens... ils étaient Chiliens, Algériens, ils étaient Espagnols et Portugais... et Africains. Ils étaient sans frontières. Ils rêvaient de révolution au Maroc et en Tunisie, dans le monde arabe et le monde entier. Ils sont partis avant le soleil. [...] Plus tard, nous venions avec les comités et les collectifs de solidarité avec les immigrés. Il y avait Djamila. Elle aussi est partie. Enfin, ces dernières années, avec nos anciens et la gauche de Colombes rassemblée. Les massacres, il y en a eu beaucoup... Durant toute la colonisation de l'Algérie : à Sétif, à Guelma et dans le Constantinois. 45000 morts un certain 8 mai 45 . Il y a eu le 17 octobre 1961 et dix ans après, celui qui voulait rendre hommage aux victimes de Charonne, Pierre Overney, fut abattu aux portes de Renault Billancourt. Venir ici chaque année ne me fait pas oublier Djillali Ben Ali, Mohammed Diab. Ne me fait pas oublier Zyed et Bouna. Avec eux, je vis les jours, les mois et les années. Avec eux, je vis chaque jour des 17 octobre 196140 ».

Au terme d'une décennie de luttes de mémoire, de 2005 à 2015, si l'oubli semble avoir localement laissé place à la reconnaissance de passés coloniaux et immigrés, les avancées nationales restent, elles, minimes. Quant au 17 octobre, le bilan officiel de deux morts et un blessé est à peine révisé ; les archives d'État, inaccessibles, épargnent toujours les plus hautes responsabilités. L'image de communes avant-gardistes contre un national frileux devant une nouvelle « histoire-problème » de l'immigration en est plutôt confortée. Mais l'entrée en scène de municipalités de " gauche » peu touchées par l'événement au moment des faits force un second constat : le nouveau gouvernement de la mémoire suit un mouvement plus complexe de renouvellement de clientèles (politiques, électorales) en banlieue, et la mobilisation d'entrepreneurs de mémoire issus des cités qui activent ces passés et la nécessité, pour les dirigeants locaux, de les recevoir ou se les approprier.

$* * *$

Sans sol pour s'arrimer, les mémoires populaires se désunissent. Pour se constituer et se transmettre, la mémoire ne peut qu'être liée à un ou des groupes qui doivent eux-mêmes disposer d'une stabilité, spatiale et temporelle, minimale. L'auteur du discours, Chérif C., en est le témoin. Rare militant des années 1970, migrateur de quartier en quartier, son militantisme s'enracine de longues années durant entre Nanterre, Colombes, Asnières et

40. Discours de Chérif C., Parc de l'Île Marrante, Colombes, 16 octobre 2011. 
Argenteuil. Sans ces conditions, l'omni-présent sans histoire efface mémoire et pensée, comme le présageait le Nous autres d'Eugène Zamiatine et, à sa suite, le 1984 de Georges Orwell. La difficulté à fixer une mémoire des lieux et des événements rejoint ici l'imagerie de banlieues en perpétuel chantier, de rénovations en démolitions, d'habitats précaires en populations en déplacement constant. "L'habitat du pauvre est volatile, celui du riche demeure ${ }^{41}$ ». La sensation redouble concernant le logement étranger : exceptionnel, temporaire, transitoire... du bidonville à la cité. À l'heure où le passé immigré est investi au point d'apparaître comme une priorité locale, un profond changement s'est donc produit : derrière le bouleversement urbain, les populations immigrées ou anciennement «immigrées » ont remplacé le cœur politique, culturel et ouvrier des cités. C'est moins le bidonville, la reproduction coloniale ou les conditions de vie qui sont visées par le message du passé institutionnalisé ; c'est avant tout la « communauté » de vie, de destin, toujours présente, la clientèle électorale qui occupe ces cités, se mobilise dans les associations, travaille dans les services de la ville. Comme hier avec la transformation du bourg agricole en ville industrielle, le changement s'attache politiquement à un bastion, populaire et immigré, surnuméraire au sein d'un parc HLM ancien, encerclé et surplombé par les tours de la Défense.

Après une longue traversée du désert des relations entre gauche et populations immigrées, l'intronisation d'une partie de la culture immigrée, de ses morts, de ses drames et de ses grandes figures apparaît bien, à Nanterre et dans les anciennes banlieues dites "rouges ", comme la conséquence de cette remarquable transformation. Le passé et les morts, scandée par certains militants de la cause de la mémoire, fournissent une ressource politique à des élus désormais sans appareil partisan d'affirmer leur changement de regard sur l'immigration, renouveler la fidélité d'un électorat populaire en se présentant à lui différemment. Les mutations sociales, économiques et les liens au présent rénovent en peu de temps le passé, déplacent le curseur des identités collectives, du communisme municipal aux drames et aux héritages des immigrations. C'est dire combien le gouvernement des morts est aussi un renouvellement par les mots au service d'une continuité représentative municipale et identitaire, sur les traces d'une autochtonie nanterrienne, d'un certain nanterrianisme.

Les usages et sélections des passés commémorés éclairent cette nouvelle gouvernance locale : les contraintes économiques restant stables sinon régies par l'austérité budgétaire, le passé offre un moyen de marquer l'ancrage «à gauche » et aux côtés « du peuple ». Comme forme de réagencement et de réajustement du pouvoir local à faible coût, la nouvelle page immigrée $d u$ communisme et $d u$ socialisme municipal accélère en retour le virage représentatif et l'intégration d'élus de l'immigration dans les conseils. La progressive méto-

41. Marc Perrone cité in Thorn J.-P., 93, La belle rebelle, Blaq Out, 2010, 73 min. 
nymie de l'immigré et du populaire exprime cette refonte plus générale du lien entre associations de quartier et pouvoir local, avec en contreplan l'ascension d'une élite intellectuelle de l'immigration mobilisée autour de son passé.

À la promotion d'une "culture immigrée ", en vogue dès le milieu des années 1980, succèdent des histoires coloniales et d'immigration longtemps regardées avec distance par les élus pour leur haut degré de conflictualité. L'histoire politique s'insinue peu à peu, à mesure qu'elle « représente » et s'adapte à l'identité du territoire, c'est-à-dire à celle que veulent bien lui donner, ou sont contraints de lui donner, les élus. Longtemps souterrains ou empruntés ${ }^{42}$, ces passés de l'immigration et de la colonisation alimentent désormais les panthéons municipaux, à gauche, comme hier les hérauts ouvriers de la Commune de Paris aux penseurs socialistes ou dirigeants de la constellation soviétique - et les résistants ornaient les rues du communisme et du socialisme municipaux.

42. House J., MacMaster N., Paris 1961..., op. cit., pp. 323-375. 
